\title{
The appearance of magnetospheric instability in flaring activity at the onset of X-ray outbursts in A0535+26
}

\author{
K. Postnov ${ }^{1,2}$, R. Staubert ${ }^{1}$, A. Santangelo ${ }^{1}$, D. Klochkov ${ }^{1}$, P. Kretschmar ${ }^{3}$, and I. Caballero ${ }^{1}$ \\ 1 Institut für Astronomie und Astrophysik, University of Tübingen, Sand 1, 72076 Tübingen, Germany \\ e-mail: Kpostnov@gmail.com \\ 2 Sternberg Astronomical Institute, 119999, Moscow, Russia \\ 3 European Space Agency, European Space Astronomy Center, PO Box 78, 28691 Villanueva de la Cañada, Madrid, Spain
}

Received 19 December 2007 / Accepted 14 January 2008

\begin{abstract}
We argue that X-ray flaring variability observed in the transient X-ray pulsar A0535+26 is due to low-mode magnetospheric instability. This instability develops at the onset of accretion, in the thin boundary layer between the accretion disk and neutron star magnetosphere. As a result, the matter collected in the boundary layer can rapidly fall onto the NS surface close to the magnetic poles, but not exactly along the field lines by which the stationary accretion proceeds. This explains the shift in cyclotron line energy measured using RXTE data in a pre-outburst spike, with respect to the line energy observed during the main outburst. Furthermore, the instability can account for the difference in pulse profiles, and their energy evolution that is different in the pre-outburst flare and main outburst.
\end{abstract}

Key words. accretion, accretion disks - stars: neutron - X-rays: binaries

\section{Introduction}

The transient X-ray binary pulsar A0535+26 was discovered by the Ariel V satellite during a giant outburst (Coe et al. 1975; Rosenberg et al. 1975). The neutron star (NS) rotates with a period $P \simeq 103 \mathrm{~s}$ and is in an eccentric $(e=0.47)$ orbit about a massive O9.7IIIe star with an orbital period of $P_{\text {orb }} \sim 110 \mathrm{~d}$ (Coe et al. 2006). The X-ray activity of this transient source is complicated: several giant outbursts were observed in 1975, 1980, 1983, 1989, 1994 and 2005, with weaker outbursts sometimes observed at successive periastron passages. This type of $\mathrm{X}$-ray activity is common for Be/X-ray binary systems, which represent the largest subclass of massive X-ray binaries. The giant (type II, according to Stella et al. 1986) outbursts can take place at any orbital phase and most probably are triggered by the enhanced activity of the optical star (Coe et al. 2006). More regular, weaker type I outbursts are associated with enhanced interaction of the neutron star with a circumstellar disk surrounding the Be-companion (Okazaki \& Negueruela 2001). A0535+26 did not show outbursting activity between 1994 and 2005. The last giant outburst of A0535+26 occurred after a prolonged quiescence period in May-June 2005 (Tueller et al. 2005). In September 2005, the subsequent normal type I outburst was extensively studied by RXTE and INTEGRAL (Caballero et al. 2007,2008 ). These observations revealed somewhat unexpected features, which we summarize below.

Feature 1. A short (fraction of a day) pre-outburst X-ray spike, with a luminosity comparable to the outburst maximum, is observed during the rise of the outburst.

Feature 2. The NS pulse period at the onset of the outburst, remained constant within the margins of error, but rapidly decreased after the periastron passage.

Feature 3. RXTE data shows that the energy of the cyclotron resonance scattering feature (CRSF) during the spike at $90 \%$ confidence is $52.0_{-1.4}^{+1.6} \mathrm{keV}$ (Caballero et al. 2008), which is notably higher than the value $46.1 \pm 0.5 \mathrm{keV}$ measured during the main outburst at the same level of X-ray luminosity. The latter value is consistent with INTEGRAL measurements (Caballero et al. 2007).

Feature 4. The CRSF energy in the main outburst (within the margins of error) is independent of the X-ray luminosity.

Feature 5. The X-ray pulse profiles during the spike are different from the pulse profiles observed during the main outburst. Pulse profiles during the outburst show an abrupt change at energies above the cyclotron resonance, while those in the preoutburst spike do not.

The additional inspection of the Swift BAT $(15-50 \mathrm{keV})$ light curve of the source (Fig. 1) reveals that the pre-outburst spike observed by RXTE is only one of a collection of flares, with the characteristic time up to a few times $10^{4} \mathrm{~s}$, appearing at the rising part of the outburst. The flux evolution then proceeds more smoothly during the spin-up of the NS (Feature 6), which begins close to periastron passage. A similar flaring activity was observed during the onset of the preceding giant outburst, at the same flux level, in April 2005, and at the next periastron passage in December 2005.

In this Letter we argue that the flaring activity and preoutburst spikes are most likely due to magnetospheric instability. This would take place at the onset of a smooth increase in the mass-accretion rate through the accretion disk surrounding the NS, close to periastron passage. This instability would cause plasma that had accumulated in the thin boundary layer, between the accretion disk and NS magnetosphere, to fall onto the NS surface as large blobs. These blobs would be channeled to regions close to the NS magnetic poles by different magnetic field lines than those guiding the accretion flow smoothly increasing from the disk. 


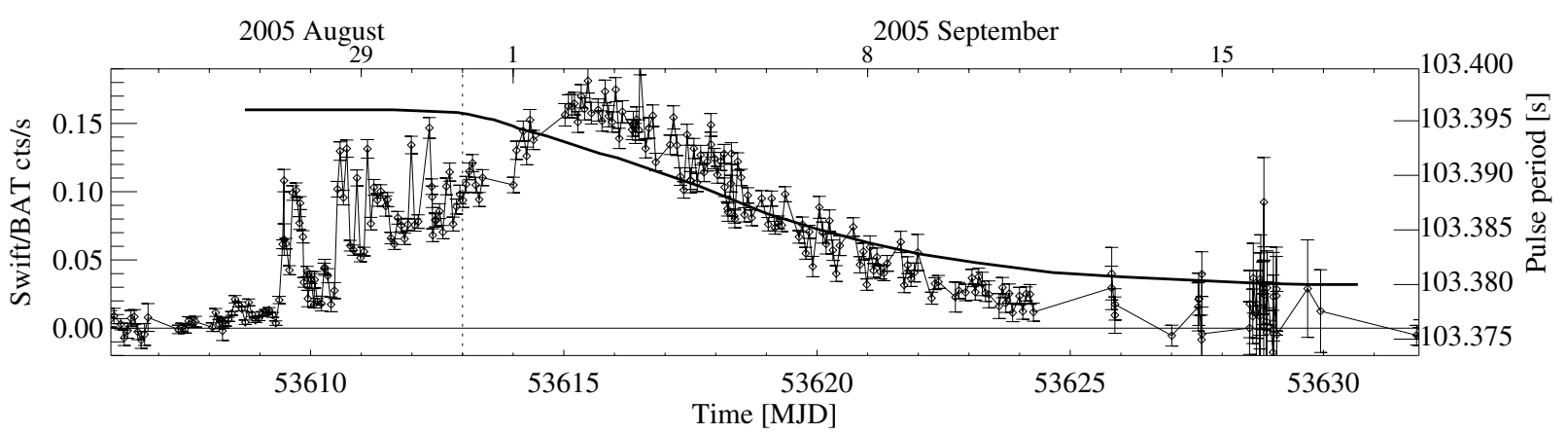

Fig. 1. The Swift/BAT light curve of A0535+26 during the normal outburst in August/September 2005. The smooth solid line shows the pulse period development as measured by RXTE (from Caballero et al. 2007b). The dashed line shows the time of periastron passage.

\section{The model}

Since the 1970 s, it has been known that matter can penetrate the NS magnetosphere, and be accreted onto magnetized NS, via various instabilities. The gravitational interchange instability (Rayleigh-Taylor, or Kruskal-Schwarzschild [KS] for plasma in magnetic field) of cold plasma at the magnetospheric boundary (see Elsner \& Lamb 1976; Arons \& Lea 1976a), in particular, mediates quasi-spherical accretion. In this case, close to the magnetic equator where KS instability is more likely, plasma enters the magnetosphere as bubbles or filaments that fall deeper towards the NS. All or part of these filaments, broken up into smaller pieces by Kelvin-Helmholtz instabilities (Arons \& Lea 1976a), can then be entrained by the magnetic field and channeled toward the magnetic polar region on the NS surface.

During disk accretion onto a rotating magnetized NS the Kelvin-Helmholtz instability between the disk material and the magnetosphere is also important (Anzer \& Börner 1980, 1983). The transition between the accretion disk and the rotating NS magnetosphere (for the aligned magnetic dipoles) was studied by Ghosh \& Lamb (1978, 1979), Scharlemann (1978), Anzer \& Börner (1983) and later by Lovelace et al. (1995) using more realistic assumptions. In the latter model, the twisting of the stellar magnetic field by the differentially rotating disk leads to the appearance of open magnetic field lines extending outward from both the star and the disk. The accretion process may then be maintained by an MHD outflow from the disk that transports away angular momentum. Numerical MHD simulations of the disk interaction with an inclined magnetic dipole (Romanova et al. 2003) confirmed the basic features of the diskmagnetospheric interaction. Accretion onto NSs can be unstable, leading to outbursts and flares, because of instabilities in the magnetosphere (Baan 1979; Spruit \& Taam 1993). The KS instability affects accretion onto magnetized NSs at both large and small accretion rates. At small accretion rates, matter accumulates at the boundary layer and flaring events are expected. At large accretion rates, matter is accreted more steadily.

In the case of A0535+26 we deal with non-stationary accretion triggered by enhanced NS interaction with the circumstellar disk and the wind of the Be-companion at binary periastron passage. BATSE observations of this source (Bildsten et al. 1997) indicated the strong disk accretion spin-up during type II (giant) outbursts, while no significant spin-up was observed during a series of type I outbursts, at successive periastron passages both before and after the giant outburst. Moreover, the average spindown of the NS rotation is clearly seen in the BATSE data before and after the giant outburst, suggesting that a propeller-like mechanism operates between accretion episodes close to periastron. We expect similar long-term spin-up/spin-down behavior of magnetized NSs in eccentric Be X-ray binaries on very general grouds (Stella et al. 1986). The magnetic propeller regime was investigated by Lovelace et al. (1999). Numerical MHD simulations (Ustyugova et al. 2006) revealed the non-stationary character of mass outflow during the propeller stage. Clearly, the transition from propeller to accretion stage is the most complicated for the analysis. Here we suggest that the non-stationary features observed in the outburst of A0535+26 relate to the magnetospheric instability which develops during the transition to the accretion stage.

The pre-outburst X-ray flaring activity is not an exceptional feature of the 2005 outbursts in A0535+26. Hints of other flares in A0535+26 are apparent for several other type I outbursts in BATSE light-curve data for its giant outburst in 1994 (see Fig. 1 of Finger et al. 1996). Similar pre-outburst spikes are observed in other Be/X-ray binaries, e.g. in BATSE observations of GS 1843-02 (Finger et al. 1999) and INTEGRAL observations of EXO 2030+375 (Camero Arranz et al. 2005). A plausible picture may be delineated as follows.

1) We assume that an accretion disk surrounds the NS in A0535+26. It is definitely the case during the giant outbursts, as the spin-up measurements and QPO observations suggest (Sembay et al. 1990; Finger et al. 1996). The disk can survive for several orbits after the giant outburst. The observed strong spin-up during the main part of the August/September 2005 outburst (Caballero et al. 2008 and Fig. 1) confirms the presence of the disk.

2) When the accretion rate onto the NS increases as it approaches orbital periastron, the magnetospheric radius $R_{\mathrm{m}}$, determined by magnetic pressure balancing plasma pressure, decreases according to the proportionality $R_{\mathrm{m}} \propto \dot{M}^{-2 / 7}$. Accretion occurs when the stopping radius of matter $R_{\mathrm{a}}$, generally of the order of $R_{\mathrm{m}}$, becomes smaller than the corotation radius $R_{\mathrm{c}}=$ $\left(G M / \omega^{2}\right)^{1 / 3}$, where $M$ is the NS mass and $\omega=2 \pi / P$ is its spin frequency. In all models of the disk-magnetospheric interaction a thin boundary layer between the disk and the rotating NS magnetosphere does exist in which the matter becomes gradually captured by the magnetic field lines. The width of this region is model dependent, but is of the order of the disk thickness (e.g., $<20 c_{\mathrm{S}} / \omega_{\mathrm{K}}$ in Anzer \& Börner 1983, or $\sim 5 c_{\mathrm{s}} / \omega_{\mathrm{K}}$ in Lovelace et al. 1995; here $c_{\mathrm{S}}$ is the sound speed and $\omega_{\mathrm{K}}$ is the Keplerian frequency). So for an estimate we can assume $\Delta l \sim h \simeq 0.1 R_{\mathrm{a}}$. The amount of mass accumulated in this layer can be roughly evaluated assuming the standard $\alpha$-theory (Shakura \& Sunyaev 1973):

$\Delta M=\rho 2 \pi R_{\mathrm{a}} 2 h \Delta l \simeq\left(4 \times 10^{19} \mathrm{~g}\right) R_{\mathrm{a}, 9}^{7 / 5} \alpha^{-4 / 5} \dot{M}_{-10}^{3 / 5}(1-\xi)^{3 / 5}$,

where $R_{\mathrm{a}, 9}$ is the stopping radius in units of $10^{9} \mathrm{~cm}, \dot{M}_{-10}$ is the mass accretion rate in units of $10^{-10} M_{\odot} / \mathrm{yr}, \xi=\dot{J} /\left(\dot{M} \sqrt{G M R_{\mathrm{a}}}\right)$ 
is the angular mometum flux through the disk normalized to the flux from the stopping radius. Here we have assumed the gas pressure to dominate over the radiation pressure in the boundary layer and the main opacity to be due to Thomson scattering. Of course, the disk is already not Keplerian at this radius $(\mathrm{d} \omega / \mathrm{d} r \rightarrow$ 0 at $R_{\mathrm{a}}$ ), but for the case of interest $R_{\mathrm{a}} \sim R_{\mathrm{c}}$ we neglect the deviation from the Keplerian motion. At the onset of accretion $\xi \simeq 0$, the viscosity parameter can be very small, $\alpha \sim 0.01$ or smaller, so the amount of matter in the boundary layer may be as high as $\sim 10^{21} \mathrm{~g}$.

3) As inferred from the analysis by Baan (1979), at small accretion rates $\left(\sim 10^{-10} M_{\odot} / \mathrm{yr}=10^{16} \mathrm{~g} / \mathrm{s}\right)$ the magnetopause in A0535+26 with $P \sim 104$ s may be on the verge of a KS instability. The model by Baan $(1977,1979)$ has sucessfully described the statistical properties and the burst waiting time luminosity correlation of type II outbursts in the rapid burster MXB 1730-335. In the linear regime, the KS instability grows with time exponentially in proportion to $\exp (\Gamma t)$ with the increment $\Gamma^{2}=k g_{\text {eff }} \tanh (k z)$ (Arons \& Lea 1976b; Baan 1979), where $k$ is the angular wave number, $z$ is the characteristic height above the interface and $g_{\text {eff }}$ is the effective gravity acceleration. $g_{\text {eff }}$ takes into account the gravitational attraction $\left(\propto 1 / R_{\mathrm{m}}^{2}\right)$, minus stabilizing contributions from the centrifugal force $\left(\propto \omega^{2} R_{\mathrm{m}}\right)$, the bending of field lines by the disk, the curvature of the magnetic field near the magnetopause $\left(\propto 1 /\left(\rho_{\mathrm{m}} R_{\mathrm{m}}^{7}\right)\right)$, possible velocity gradients in the plasma flow, etc. The condition for the instability to occur is the change of the sign of $g_{\mathrm{eff}}$ from negative (stability) to positive (instability), which defines the zero-surface $g_{\text {eff }}\left(R_{z}\right)=0$ at some distance from the NS. We note that under the assumption of the corotation of matter and the magnetosphere, the instability can occur only when the zero-surface is inside the corotation radius, $R_{z}<R_{\mathrm{c}}$, because of the centrifugal term in $g_{\mathrm{eff}}$. This implies that instability can develop only when accretion is permitted centrifugally. This is consistent with the observation in A0535+26 of flaring activity, and accretion.

As the magnetopause moves inwards through the zerosurface with increasing $\dot{M}$, the simplified analytical treatment by Baan (1979) shows that the lowest harmonics are the first to become unstable. This finding however remains controversial. Early numerical simulations of the disk-magnetosphere interaction (e.g. Rastätter \& Schindler 1999) have shown that the high- $m$ modes grow faster. The contribution of the $B_{\phi}$ component of the field in the disk can suppress high- $m$ modes. Simulations by Wang \& Nepveu (1983) have shown that the high- $m$ modes grow faster but may later merge, forming larger filaments. The first global 3D MHD simulations of the disk-magnetosphere interaction through the KS instability (Romanova et al. 2007) have shown that the low- $m$ modes dominate for a wide range of parameters. It is not however clear which factors are responsible for suppressing the high- $m$ modes. These 3D simulations have shown that at small accretion rates matter accretes through funnel streams, while at large accretion rates, matter accretes through gravitational KS instabilities. The 3D simulations have been carried out for quite small magnetospheres $\left(\sim 10 R_{\mathrm{NS}}\right)$ which are more applicable for the millisecond pulsars. This is probably why the accumulation of matter at small $\dot{M}$ and bursting events have not yet been observed in these simulations. Therefore, we can assume that for Be/X-ray transients with large magnetospheres, such as A0535+26, lowmode magnetospheric instability, initiated by an increase in $\dot{M}$, can develop.

In such binary systems the infall of plasma blobs from the magnetopause can also be triggered by variation of the rate of mass captured from the variable stellar wind. So short X-ray flares or spikes can in principle be observed at any orbital phase. An "unexpected" X-ray flare in A0535+25 observed by INTEGRAL in October 2003 during the prolonged quiescence period (Hill et al. 2007) could be an example of such an event.

4) The low-mode magnetospheric instability can break-up the entire boundary layer in the disk. All material stored in the boundary layer may then rapidly enter the magnetosphere. Our estimate above indicates that up to $\sim 10^{21} \mathrm{~g}$ can fall onto the NS surface on the free-fall time scale (of the order of $20 \mathrm{~s}$ ) producing short X-ray spikes with a maximum X-ray luminosity of up to $\sim 5 \times 10^{36} \mathrm{erg} / \mathrm{s}$ (feature 1 from the Introduction). The amount of matter falling onto the NS surface during the spikes in the considered outburst of A0535+26 can be estimated from the light curve (Fig. 1) and is found to be $\Delta M \simeq \int \dot{M} \mathrm{~d} t \lesssim 10^{21} \mathrm{~g}$ in each spike. The characteristic timescale of the spikes is $\sim 10^{4} \mathrm{~s}$ and agrees with the time required to replenish the boundary layer $\sim \Delta M / \dot{M}$. The angular momentum supply from the disk during flares changes the NS spin period by the fractional amount $|\Delta P / P|=\Delta M \sqrt{G M R_{\mathrm{a}}} P /(2 \pi I) \lesssim 7 \times 10^{-6}$ where we assume that the NS moment of inertia is $I=10^{45} \mathrm{~g} \mathrm{~cm}^{2}$ and $R_{\mathrm{a}}=R_{\mathrm{c}}=$ $10^{9} \mathrm{~cm}$. This is within the uncertainty of the period measurement $\left(\triangle P / P \sim 5 \times 10^{-6}\right.$, Caballero et al. 2008), which explains the lack of noticeable spin-up of the NS at the initial phase of the outburst (feature 2 from the Introduction); see also the BATSE observations of A0535+26 presented in Bildsten et al. (1997), and of GS 1843-02 in Finger et al. (1999).

5) The plasma entering the magnetosphere via the low-mode $\mathrm{KS}$ instability may become frozen into the magnetic field more close to the NS than the main accretion flux and hence fall along different magnetic field lines than those by which the quasi-stationary accretion is channeled. This explains the observed difference in the cyclotron line energy during the initial $\mathrm{X}$-ray spike and the remainder of the outburst (feature 3 from the Introduction). Similarly to Her X-1 (Staubert et al. 2007), the absence of a radiation-dominated accretion column in A0535+26 is suggested by the independence of the CRSF energy late in the outburst from the observed X-ray luminosity (feature 4 from the Introduction). The height of the emission region above the NS surface is then about several hundred meters. To change the CRSF energy by the fractional amount of $\Delta E_{\mathrm{c}} / E_{\mathrm{c}} \sim 10 \%$, the emission region during the spike is required to move towrds the NS surface by the amount $\Delta R / R_{\mathrm{NS}} \sim 3 \%$ (assuming the dipole magnetic field), i.e. by about 300 meters. Therefore, the emission from the spike is likely to originate very close to the NS surface. This explains the different pulse profiles during the spike, especially in hard X-rays (feature 5 from the Introduction).

The smooth change in pulse profile shape at energies above the cyclotron resonance (as in Her X-1, Klochkov et al. 2008) may correspond to a pencil-beam diagram of emission during the flare. The photon cross-section may then change smoothly, at the cyclotron resonance energy, in the strong magnetic field along the direction of the field. In the main outburst, however, the accretion column is higher (see above), the density increases such that it can become optically thick in the direction normal to the field, and if there is a temperature gradient to the center, a fan-like beam can be additionally formed by extraordinary photons ${ }^{1}$. The cross-sections of extraordinary photons far from the resonance $\left(E \ll E_{\mathrm{c}}\right)$ are proportional to $\sigma_{\perp} \simeq \sigma_{\mathrm{T}}\left(E / E_{\mathrm{c}}\right)^{2}$, which is a far smaller cross-section than that of ordinary

\footnotetext{
1 Ordinary polarized photons have electric field vector $\boldsymbol{E}$ lying in the plane formed by the magnetic field and the wave vector of the photon; the $\boldsymbol{E}$-vector of extraordinary photons is perpendicular to this plane.
} 
photons $\sigma_{\|} \simeq \sigma_{\mathrm{T}}\left(\sin ^{2} \theta+\cos ^{2} \theta\left(E / E_{\mathrm{c}}\right)^{2}\right)$. The e-photons are then able to escape effectively from larger optical depth. (Here $\sigma_{\mathrm{T}}$ is the Thomson scattering cross-section and $\theta$ is the angle between the incident photon and the magnetic field; see Harding \& Lai $2006^{2}$.) Above cyclotron resonance, $\sigma_{\perp} \simeq \sigma_{\|} \simeq \sigma_{\mathrm{T}}$ and photons escape from small optical depths. This can explain the disappearance of the large hump at pulse phase $\sim 0.7$ after crossing the CRSF energy (Fig. 3 in Caballero et al. 2008).

6) As the accretion rate increases, the NS starts spinningup and the angular momentum flux through the inner disk rapidly approaches its maximum possible value $\sim \dot{M} \sqrt{G M R_{\mathrm{a}}}$ (see Caballero et al. 2008 and Fig. 1). The parameter $\xi$ in the expression for $\Delta M$ above increases from zero to almost one, which strongly reduces the mass in the disk boundary layer and hence the mass available for unstable accretion. The amplitude of spikes rapidly decreases and we do not see strong flaring activity during the spin-up stage (feature 6 from the Introduction); see also Figs. 2 and 10 in Finger et al. (1999) for the case of GS 1843-02. This consideration suggests that flares can reappear after the spin-up stopping, for which there is indeed a hint in the light curve of the A0535+26 outburst during September 13-15, 2005. At this time the NS had stopped spinning-up (see Fig. 1).

\section{Discussion}

The magnetospheric instability model proposed for the preoutburst flares in A0535+26 is generic and can be applied to other transients. The prerequisite, however, is that the source must be on the verge of low-mode instability, which depends on the NS magnetic field, the spin period, the accretion rate and possibly other parameters (e.g. the misalignment of the magnetic dipole and/or NS spin axis relative to the orbital angular momentum). It is possible that the transition from propeller to accretion stage can occur without a strong KS instability being observed. The model predicts changes in pulse profiles and in the cyclotron line energy during the short flares, as observed in A0535+26 (Caballero et al. 2008), which can be compared with observations of other sources.

Are there other possible explanations for the observed preoutburst X-ray spikes?

The SPH modeling of an accretion disk surrounding the NS (Hayasaki \& Okazaki 2006) reproduces normal outbursts at successive periastron passages. In some instances the modeling shows a single peak preceding outburst maximum. The accretion disk is formed from the Roche lobe overflow of the coplanar circumstellar disk surrounding the Be companion star. The important feature of these SPH simulations is the transient, one-armed spiral structure of the accretion disk, induced by a phase-dependent mass accretion. Such accretion disks can be responsible for mass transfer enhancement close to periastron. The model by Hayasaki \& Okazaki, however, ignores the disk-magnetosphere interaction critical to transient accretion onto magnetized NS, and can not reproduce the observed flaring activity.

Spruit \& Taam (1993) also found a viscous, diskmagnetosphere instability that was associated with the magnetospheric boundary around the corotation radius. This instability results in a cyclic enhancement of the mass accretion rate on the viscous time scale at the magnetospheric boundary. While this timescale can be as short as $10^{4} \mathrm{~s}$ for the corotation radius
$10^{9} \mathrm{~cm}$, the chaotic behaviour of flares observed in A0535+26 and, most importantly, other features (different pulse profiles and CRSF energy, and absence of the flaring during the NS spinup phase) imply that this model can not provide the correct explanation.

Our model for short flares observed at the onset of outbursts in the transient X-ray pulsar A0535+26 is based on magnetospheric instability. It succesfully explains all features of the outburst observed during August-September 2005 by RXTE and INTEGRAL, and makes clear predictions for future observations. The present-day accuracy of cyclotron line measurements in accreting neutron stars, precise timing analysis and evolution of X-ray pulse shapes with luminosity at different energies, have became the working tools to probe the non-stationary accretion onto magnetized neutron stars.

Acknowledgements. The authors acknowledge H. Spruit, F. Meyer, R. Sunyaev and V. Suleimanov for useful discussions. K.A.P. thanks the staff of the IAAT for hospitality and grants DAAD A/07/09400 and RNP-2.1.15940 for support. Research has made use of Swift/BAT transient monitor results provided by the Swift/BAT team.

\section{References}

Anzer, U., \& Börner, G. 1980, A\&A, 83, 133

Anzer, U., \& Börner, G. 1983, A\&A, 122, 73

Arons, J., \& Lea, S. 1976a, ApJ, 207, 914

Arons, J., \& Lea, S. 1976b, ApJ, 210, 792

Baan, W. A. 1977, ApJ, 214, 245

Baan, W. A. 1979, ApJ, 227, 987

Bildsten, L., Chakrabarrty, D., Chiu, J., et al. 1997, ApJS, 113, 367

Caballero, I., Kretschmar, P., Santangelo, A., et al. 2007, A\&A, 465, L21

Caballero, I., Santangelo, A., Kretschmar, P., et al. 2008, A\&A, 480, L17

Camero Arranz, A., Wilson, C. A., Connell, P., et al. 2005, A\&A, 441, 261

Coe, M. J., Carpenter, G. F., Engel, A. R., \& Quenby, J. J. 1975, Nature, 256, 630

Coe, M. J., Reig, P., McBride, V. A., Galache, J. L., \& Fabregat, J. 2006, MNRAS, 368, 447

Elsner, R. F., \& Lamb, F. K. 1976, Nature, 262, 336

Finger, M., Wilson, R. B., \& Harmon, B. A. 1996, ApJ, 459, 288

Finger, M., Bildsten, L., Chakrabarty, D., et al. 1999, ApJ, 517, 449

Ghosh, P., \& Lamb, F. K. 1978, ApJ, 223, L83

Ghosh, P., \& Lamb, F. K. 1979, ApJ, 232, 259

Harding, A., \& Lai, D. 2006, Rep. Progr. Phys., 69, 2631

Hayasaki, K., \& Okazaki, A. T. 2006, MNRAS, 372, 1140

Hill, A. B., Bird, A. J., Dean, A. J., et al. 2007, MNRAS, 381, 1275

Kaminker, A. D., Pavlov, G. G., \& Shibanov, Yu. A. 1983, Ap\&SS, 91, 167

Klochkov, D. K., Staubert, R., Postnov, K., et al. 2008, A\&A, in press

Lovelace, R., Romanova, M., \& Bisnovaty-Kogan, G. S. 1995, MNRAS, 275, 244

Lovelace, R., Romanova, M., \& Bisnovaty-Kogan, G. S. 1999, ApJ, 514, 368

Okazaki, A. T., \& Negueruela, I. 2001, A\&A, 377, 161

Rastätter, L., \& Schindler, K. 1999, ApJ, 524, 361

Romanova, M. M., Ustyugova, G. V., Koldoba, A. V., Wick, J. V., \& Lovelace, R. V. E. 2003, ApJ, 595, 1009

Romanova, M. M., Kulkarni, A. K., \& Lovelace, R. V. E. 2007

[arXiv: 0711.0418]

Rosenberg, F. D., Eyles, C. J., Skinner, K., \& Willmore, A. P. 1975, Nature, 256, 628

Scharlemann, E. T. 1978, ApJ, 219, 617

Sembay, S., Schwartz, R. A., Orwig, L. E., Dennis, B. R., \& Davies, S. R. 1990, ApJ, 351, 675

Shakura, N. I. \& Sunyaev, R. A. 1973, A\&A, 24, 337

Spruit, H. C., \& Taam, R. E. 1993, ApJ, 402, 593

Staubert, R., Shakura, N. I., Postnov, K. A., et al. 2007, A\&A, 465, L25

Stella, L., White, N. E., \& Rosner, R. 1986, ApJ, 308, 669

Tueller, J., Ajello, M., Barthelmy, S., et al. 2005, Astron. Telegram, 504

Ustyugova, G. V., Koldoba, A. V., Romanova, M. M., \& Lovelace, R. V. E. 2006, ApJ, 646, 304

Wang, Y.-M., \& Nepveu, M. 1983, A\&A, 118, 267

\footnotetext{
2 A similar dependence holds for free-free absorption cross-sections (Kaminker et al. 1983).
} 